\section{REMOVABLE PROSTHODONTICS}

\section{A randomized clinical trial comparing anatomic, lingualized, and zero-degree posterior occlusal forms for complete dentures}

Sutton AF, McCord JF J Prosthet Dent 2007; 97: 292-298

'Flat-cusped' teeth appeared to give patients less satisfaction.

Studies of patient preferences for the occlusal form of posterior teeth have given conflicting results. In this trial, 45 patients were each asked to give their opinions on 3 different sets of complete dentures, processed as far as possible to be identical in all except posterior tooth form. The sets were: anatomical (A), lingualized (L: maxillary lingual cusps articulated with mandibular occlusal surfaces in centric working and nonworking positions), and 0-degree teeth ( $\mathrm{Z}$ ).

Patient satisfaction was assessed with a version of the Oral Health Impact Profile (OHIP-EDENT). On a variety of responses, $A$ and $L$ designs were both significantly better than $\mathrm{Z}$, and 'inability to eat' was reported for $\mathrm{Z}$ more frequently than $\mathrm{A}(P=0.05)$ and $\mathrm{L}(P=0.02)$. A and $\mathrm{L}$ did not differ.

\section{DOI: 10.1038/bdj.2007.999}

\section{PREVENTIVE DENTISTRY; CARIOLOGY}

\section{Noninvasive control of dental caries} in children with active initial lesions.

\section{A randomized clinical trial}

Hausen H, Seppä L et al. Caries Res 2007; 41: 384-391

DMFS increment was reduced in caries-active children in an area with low caries experience.

Despite a decline in caries from 1970 to 1990 , about 1/2 of Finnish 12-year-olds have had cavitational caries. Unfortunately, preventive RCTs have not reduced caries in those at high risk, perhaps because other community groups, hence social factors, had not been targeted as well. In this study, 577 11-12year-olds with active caries participated in a preventive RCT in a small town. Oral health was also actively promoted in the whole town.

After 4 yrs, 250 subjects remained in the experimental group (E), who had been given counselling and specific preventive measures, and 247 in the control group (C), who received customary dental care. After 2 yrs, DMFS increment was 1.86 for E and 2.44 for $C(P=0.02)$ and after 4 yrs, respectively 2.56 and $4.6(P<0.0001)$. The authors note that they were not able to control harmful snacking in E group.

\section{CONSERVATIVE DENTAL SURGERY}

\section{Long-term survival of complete crowns, fixed dental prostheses, and cantilever fixed prostheses with posts and cores on root canal-treated teeth}

De Backer, Van Maele et al. Int J Prosthodont 2007; 20: 229-234

There were more failures of larger bridges and of cantilevered bridges when post-and-core abutments were used.

The expected survival of endodontically treated teeth used as abutments is important when planning bridges. In this study, records for 1,037 crowns, 134 3-unit bridges, 322 bridges and 168 cantilever bridges, made 16-20 years previously, were available from a Belgian undergraduate clinic. A strength of the study was that all treatment had been according to the same principles. They represented $80 \%$ of the totals made in the clinic during the period, except for cantilevers (64\%).

Comparing vital and non-vital teeth, 18 yr survival rates for crowns were respectively 75\% and 79\% (NS). For 3-unit bridges, 15-yr survival rates were $83 \%$ and $76 \%$ (NS); for all bridges they were $84 \%$ and $64 \%(P<0.01)$; for cantilever bridges, vital group survival was 74\% at $16 \mathrm{yrs}$, and non-vital survival was $52 \%$ at 18 yrs $(P<0.01)$.

DOI: $10.1038 /$ bdj.2007.1000

ONCOLOGY; BEHAVIOURAL SCIENCE

An assessment of deprivation as a factor in the delays in presentation, diagnosis and treatment in patients with oral and oropharyngeal squamous cell carcinoma

Rogers SN, Pabla R etal. Oral Oncol 2007; 43: 648-655

Professional delay was less for more advanced tumours and for the most deprived areas.

Patients with oral and oropharyngeal carcinoma frequently do not present until disease is advanced. Data were reviewed from 559 patients seen over an $11 \mathrm{yr}$ period. About 60\% were smokers and $1 / 3$ were heavy drinkers. About half presented to medical practitioners and half to dentists.

Median times in days were: for referral letter to specialist unit, 0 (IQR 0-0); to biopsy result, 22 (9-51); to operation 47 (29-75); from definitive diagnosis to definitive treatment, 21 (12-30). After analyis, the authors concluded that deprivation in their patients led to quicker attention, possibly because of proximity to large teaching hospitals.

DOI: $10.1038 /$ bdj.2007.1002 